\title{
Comparative genome analysis of an avirulent and two virulent strains of avian Pasteurella multocida reveals candidate genes involved in fitness and pathogenicity
}

Timothy J Johnson ${ }^{1 *}$, Juan E Abrahante' ${ }^{1}$, Samuel S Hunter ${ }^{2}$, Melissa Hauglund ${ }^{3}$, Fred M Tatum³, Samuel K Maheswaran ${ }^{1}$ and Robert E Briggs ${ }^{3}$

\begin{abstract}
Background: Pasteurella multocida is the etiologic agent of fowl cholera, a highly contagious and severe disease of poultry causing significant mortality and morbidity throughout the world. All types of poultry are susceptible to fowl cholera. Turkeys are most susceptible to the peracute/acute forms of the disease while chickens are most susceptible to the acute and chronic forms of the disease. The whole genome of the Pm70 strain of P. multocida was sequenced and annotated in 2001. The Pm70 strain is not virulent to chickens and turkeys. In contrast, strains X73 and P1059 are highly virulent to turkeys, chickens, and other poultry species. In this study, we sequenced the genomes of $P$. multocida strains X73 and P1059 and undertook a detailed comparative genome analysis with the avirulent Pm70 strain. The goal of this study was to identify candidate genes in the virulent strains that may be involved in pathogenicity of fowl cholera disease.
\end{abstract}

Results: Comparison of virulent versus avirulent avian P. multocida genomes revealed 336 unique genes among the P1059 and/or X73 genomes compared to strain Pm70. Genes of interest within this subset included those encoding an L-fucose transport and utilization system, several novel sugar transport systems, and several novel hemagglutinins including one designated PfhB4. Additionally, substantial amino acid variation was observed in many core outer membrane proteins and single nucleotide polymorphism analysis confirmed a higher dN/dS ratio within proteins localized to the outer membrane.

Conclusions: Comparative analyses of highly virulent versus avirulent avian $P$. multocida identified a number of genomic differences that may shed light on the ability of highly virulent strains to cause disease in the avian host, including those that could be associated with enhanced virulence or fitness.

Keywords: Pasteurella multocida, Genomics, Genome, Virulence, Avian, Fitness, Fowl cholera, Pathogenicity

\section{Background}

Avian pasteurellosis, also known as fowl cholera is a highly contagious, systemic, and severe disease affecting wild and domestic birds frequently resulting in high mortality and morbidity. The disease is of major economic importance throughout the world in areas of domestic poultry production [1-3]. The causative agent

\footnotetext{
*Correspondence: joh04207@umn.edu

'Department of Veterinary and Biomedical Sciences, University of Minnesota, St. Paul, Minnesota, USA

Full list of author information is available at the end of the article
}

of fowl cholera is Pasteurella multocida, a Gramnegative bacterium. Carter $[4,5]$ identified five capsular types of $P$. multocida based on differences in capsular antigens and designated them as $\mathrm{A}, \mathrm{B}, \mathrm{D}, \mathrm{E}$, and $\mathrm{F}$ serogroups. Heddleston and co-workers classified the bacterium into 16 somatic types based on differences in the lipopolysaccharide antigens [6]. In 1981, a standard system for identifying serotypes of $P$. multocida was recommended that combined both the Carter capsular typing and Heddleston somatic typing systems to designate serotypes [7] and a serotype is designated by its

\section{Biomed Central}


capsular type followed by the somatic type. Using this system, the most common serotypes causing fowl cholera in the United States are A:1, A:3, and A:3.4 [8]. While there are no indications that any particular serotype is more or less virulent than others the virulence of avian isolates of most common serotypes appears to vary considerably [9].

Fowl cholera disease can occur in peracute/acute and subacute/chronic forms [10]. All types of poultry are susceptible to the disease, although among them turkeys, pheasants and partridges are highly susceptible to peracute/acute forms of disease whereas chickens are relatively more resistant [11]. In chickens, the most common forms of the disease are acute and chronic. In peracute/acute disease there is sudden death due to terminal - stage bacteremia and endotoxic shock $[1,3]$. Signs of acute cholera have been reproduced by injection of endotoxin from P. multocida [12-14]. Post-mortem findings are dominated by general septicemic lesions. $[1,2]$. In chronic disease, signs are principally due to localized infections of leg or wing joints, comb, wattles and subcutaneous tissue of the head $[2,10]$. The completed genome of P. multocida strain Pm70 has been available for over eleven years [15] and has greatly facilitated subsequent genomic-based approaches towards better understanding the underlying genetic mechanisms related to virulence and fitness. This complete genome sequence has been used in the study of specific enzymes [16], microarray analyses of differentially expressed genes [17-20], proteomic analyses [21,22], study of virulence factors [16,23-25], reverse vaccinology approaches [26], and as a reference for assembly and comparison to other genomes. While the Pm70 genome sequence has been a great asset in our studies, progress has been modest in the identification and understanding of $P$. multocida virulence [27]. Even today, very little is known about the totality of the mechanisms behind $P$. multocida's ability to cause disease. The Pm70 strain was isolated from the oviduct of a layer chicken in 1976 from Texas (personal communication- RE. Briggs). This strain belongs to serotype F:3 [28] and not A:3 as reported earlier [15], is avirulent and does not cause experimental fowl cholera disease in chickens [28]. In contrast, other strains of $P$. multocida have been isolated, such as strains X73 and the P1059, that are highly virulent to chickens, turkeys, and other poultry species $[29,30]$.

Additional P. multocida strains of bovine, avian, and porcine origin have recently been sequenced, which was the subject of a recent comparative review [31]. The authors noted, based on the nine genomes sequenced to date, there was "no clear correlation between phylogenetic relatedness and host predilection or disease". Information is sparse on the location and characterization of the genes responsible for differences in virulence of avian and other P. multocida. In another recent review, Boyce et al. [32] speculated that the combination of additional $P$. multocida genome sequences and advances in our ability to genetically manipulate the organism will facilitate major advances in our understanding of disease pathogenesis. To that end, we undertook a detailed comparative genome analysis of two virulent strains (X73 and P1059) and avirulent strain Pm70 of P. multocida. The goal of this study is to enable narrowed identification of a repertoire of unique genes present in the highly pathogenic avian strains that may play a role in virulence. This information will also facilitate the design of improved modified live vaccine candidates with defined mutations that can be evaluated as immunoprophylactic agent(s) to control $P$. multocida-caused disease in avian and other host species.

\section{Methods}

\section{Bacterial strains}

The strains sequenced in this study included $P$. multocida strains P1059 (ATCC\# 15742) and X73 (ATCC\# 11039). Strain P1059 is a well characterized pathogenic strain isolated from the liver of a turkey that died of fowl cholera [30]. Strain X73 is also a well characterized pathogenic strain isolated from the liver of a chicken that died of fowl cholera [30]. For comparative purposes, the avirulent Pm70 strain was used [15]. There are several reasons why we selected strains P1059 and X73 in this study. First, both strains are highly virulent to chickens, turkeys and other poultry species. Second, they are of different serotypes (P1059 = A:3; and X73 = A:1) and different immunologic types [30]. Thirdly, they are reference serotype strains that are readily available to investigators and there is abundant literature on the biology of these two strains [1,11,30,33-35].

\section{Genome sequencing and annotation}

Sequencing of strains P1059 and X73 was performed using 454 Life Sciences pyrosequencing at the National Animal Disease Center in Ames, Iowa. The following data sets were generated for each strain: GS- FLX, with 270,010 shotgun reads of average length 240 bp yielding $64,827,159$ bp for P1059; and GS-FLX, with 227,030 shotgun reads of average length of $240 \mathrm{bp}$, yielding $54,398,540$ bp for X73. Reads were de novo assembled into scaffolds using Newbler 2.3 [36]. The draft sequences of these genomes are deposited under the following accession numbers: P1059 [Genbank:AMBQ0 1000000] and X73 [Genbank:AMBP01000000].

\section{Comparative genomics}

Annotation of P1059 and X73 was performed using publicly available tools. Putative coding regions were predicted using GeneMarkS [37]. Gene function was 
assigned using HMMER3 against Pfam-A 24.0, RPSBLASTp against CDD, and BLASTp against all microbial proteins [38,39]. tRNA genes were identified using tRNAscan-SE [40]. rRNA genes were identified using RNAmmer [41]. For analysis of the shared and unique proteins in the $P$. multocida genomes sequenced, BlastP was used with a similarity cutoff of $90 \%$ identity over $90 \%$ of the protein as an arbitrary designation for similar versus dissimilar proteins. For genomic island analysis, whole genome alignments were performed using MAUVE to identify regions present in strains P1059 and X73 but absent from strain Pm70 [42]. Linear and circular genomic maps were generated using XPlasMap and Circos [43]. Single nucleotide polymorphism (SNP) analysis was performed using SNPeff [44].

\section{Results and discussion}

Overview of the $P$. multocida $\mathrm{P} 1059$ and $\mathrm{X} 73$ genomes

A total of 270,010 reads were used to draft assemble strain P1059, resulting in a single scaffold of 27 large contigs (> $500 \mathrm{bp}$ ) of approximately 27-fold coverage and an estimated genome size of $2.4 \mathrm{Mb}$. A total of 227,030 reads were used to draft assemble strain $\mathrm{X} 73$, resulting in 17 large contigs (> $500 \mathrm{bp}$ ) of approximately 23-fold coverage and an estimated genome size of approximately $2.4 \mathrm{Mb}$. No plasmids were identified in either strain sequenced. The contigs generated were then aligned to strain Pm70 to generate collinear draft sequences and subsequently compare the three avian source genomes.

\section{Unique regions of virulent avian $P$. multocida}

The draft genomes of strains P1059 and X73 were found to contain 2,144 and 2,085 predicted proteins, respectively. Along with strain Pm70, the genomes all contained 51 tRNA-carrying genes and 4 rRNA-carrying operons. The genomes of the three avian $P$. multocida strains contained a remarkably high number of shared proteins $(1,848)$, which comprised $86.2-90.7 \%$ of the predicted proteins of the three avian strains using a BlastP similarity cut-off of $90 \%$ (Figure 1). Compared to strain Pm70, a total of 336 unique proteins were identified in either strains P1059 or X73, of which 61 were contained within both genomes (Table 1). Most of the 61 shared proteins were small predicted proteins of unknown function and located individually throughout the $P$. multocida genome that could be attributed to differences in annotation approaches (Figure 2). Also, most of the predicted proteins identified were present in one or more sequenced $P$. multocida from the NCBI database that were not from avian hosts. However, one noteworthy region of difference shared by P1059 and X73, but absent from Pm70 and other strains of non-avian source, was located between the core genes $\operatorname{deo} C$ and $r f a D$ in both P1059

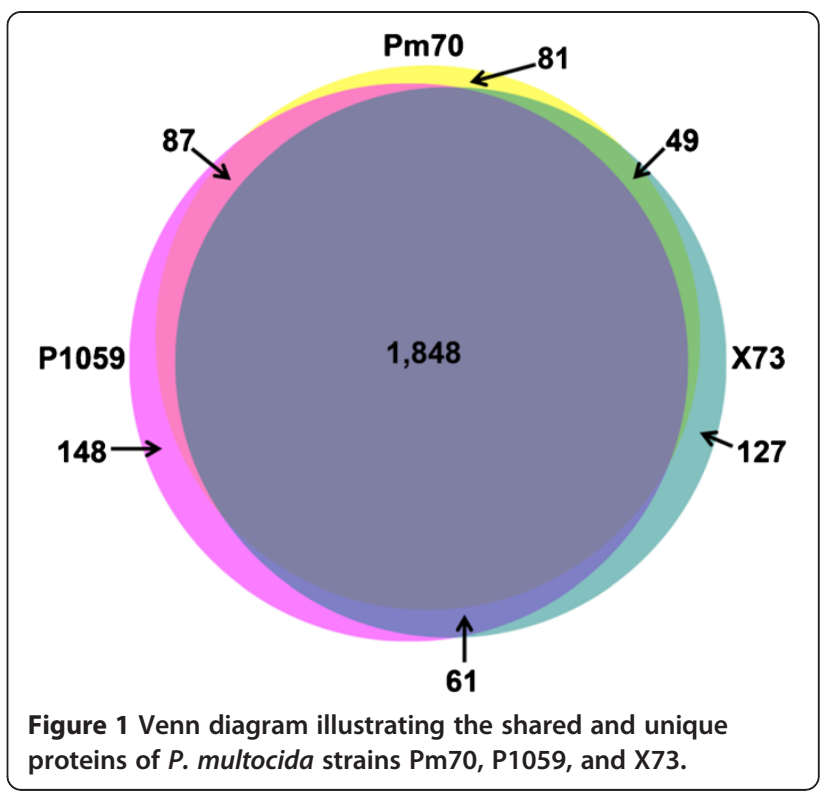

and X73 (P1059 - 01496 to 01503; X73 - 01400 to 01407). This region contained ten predicted proteins with similarity to systems involved in the transport and utilization of L-fucose. L-fucose is an important component of host mucin and has shown to be a chemoattractant for certain bacterial species, such as Campylobacter jejuni. Moreover, the ability to utilize L-fucose by C. jejuni has been shown to confer a fitness advantage for avian strains in low nutrient environments such as the respiratory tract $[45,46]$. Comparison of available $P$. multocida sequences suggests that the presence of this region may be a defining feature of pathogenic avian-source $P$. multocida strains, as it was present in P1059, X73, and P. multocida subsp. gallicida strain Anand1 isolated from a chicken in India [47] but absent from strains Pm70, pathogenic bovine-source strain 36950 [48] and pathogenic swine source strains 3480 and HN06 [49]. Other studies have demonstrated an ability of avian-source P. multocida to ferment L-fucose, further suggesting that the majority of avian-source P. multocida strains harbor this system $[9,33,50]$. Other bacteria inhabiting the respiratory tracts of poultry have been identified to utilize L-fucose, such as Gallibac terium anatis, suggesting that such capabilities may be advantageous for respiratory bacterial pathogens of poultry [51]. Such systems could play a role in increased fitness and/or virulence capability of strains P1059 and $\mathrm{X} 73$ in the avian host.

Twelve proteins were also identified that were present in both strains P1059 and X73 at greater than 90\% amino acid similarity, but at less than $90 \%$ similarity in strain Pm70 (Table 2). Among the twelve proteins identified were several membrane-associated proteins, including LspB, PfhB3, Opa, and SprT. The presence of 
Table 1 Predicted proteins of interest present in P. multocida strains P1059 and X73 at greater than $90 \%$ similarity but absent from strain Pm70

\begin{tabular}{|c|c|c|c|c|c|c|c|c|c|}
\hline \multirow[b]{2}{*}{$\begin{array}{l}\text { Gene locus } \\
\text { (P1059) }\end{array}$} & \multirow[b]{2}{*}{$\begin{array}{l}\text { Length } \\
\text { (aa) }\end{array}$} & \multirow[b]{2}{*}{$\begin{array}{l}\text { Genomic } \\
\text { island }\end{array}$} & \multirow[b]{2}{*}{ Predicted function } & \multicolumn{6}{|c|}{ Presence in: } \\
\hline & & & & $\mathrm{Pm} 70$ & P1059 & $\mathrm{X73}$ & 36950 & HN06 & 3480 \\
\hline 00226 & 66 & NA & Hypothetical protein & - & + & + & - & + & + \\
\hline 00545 & 68 & NA & Hypothetical protein & - & + & + & - & + & + \\
\hline 00580 & 828 & 12 & Trimethylamine-N-oxide reductase & - & + & + & + & + & + \\
\hline 00581 & 371 & 12 & Cytochrome c-type protein TorY & - & + & + & + & + & + \\
\hline 00881 & 1125 & 15 & Putative Ton-B dependent heme receptor & - & + & + & - & - & - \\
\hline 00948 & 62 & NA & Hypothetical protein & - & + & + & + & + & + \\
\hline 01347 & 332 & 26 & Putative DNA-binding protein & - & + & + & + & + & + \\
\hline 01412 & 52 & NA & Hypothetical protein & - & + & + & + & + & + \\
\hline 01496 & 249 & 28 & L-fucose operon activator & - & + & + & - & - & - \\
\hline 01497 & 586 & 28 & L-fucose isomerase & - & + & + & - & - & - \\
\hline 01498 & 495 & 28 & L-fuculokinase & - & + & + & - & - & - \\
\hline 01499 & 144 & 28 & L-fucose mutarotase & - & + & + & - & - & - \\
\hline 01500 & 215 & 28 & L-fuculose phosphate aldolase & - & + & + & - & - & - \\
\hline 01501 & 508 & 28 & Ribose ABC transport system, ATP-binding protein & - & + & + & - & - & - \\
\hline 01502 & 342 & 28 & Ribose $A B C$ transport system, permease protein & - & + & + & - & - & - \\
\hline 01503 & 318 & 28 & $\begin{array}{l}\text { Ribose } A B C \text { transporter, periplasmic ribose-binding } \\
\text { protein }\end{array}$ & - & + & + & - & - & - \\
\hline 01505 & 480 & 28 & Aldehyde dehydrogenase A & - & + & + & - & - & - \\
\hline 01550 & 384 & 31 & Flavohemoprotein & - & + & + & + & - & + \\
\hline 01587 & 53 & NA & Hypothetical protein & - & + & + & + & + & + \\
\hline 01686 & 108 & NA & HigA antitoxin protein & - & + & + & - & + & - \\
\hline 01825 & 60 & NA & Hypothetical protein & - & + & + & + & + & + \\
\hline 01854 & 51 & NA & Hypothetical protein & - & + & + & - & - & + \\
\hline 01963 & 52 & NA & Hypothetical protein & - & + & + & + & + & + \\
\hline
\end{tabular}

Presence of these proteins in additional sequenced P. multocida is also presented.

divergent protein sequences that are membraneassociated is suggestive of adaptation of $P$. multocida strains towards particular hosts.

There were also predicted proteins identified as unique to strains P1059 (148 total) and X73 (127 total) compared to strain Pm70. Many of these proteins were again of unknown function and/or associated with prophagelike elements (Additional file 1: Table S1 and Additional file 2: Table S2). However, some systems unique to each strain were noteworthy. In strain P1059, one unique region contained six genes predicted as involved in the transport and modification of citrate, and the conversion of citrate to oxaloacetate via citrate lyase (00080 to 00085). This system was absent in all other sequenced $P$. multocida genomes. The conversion of citrate to oxaloacetate is linked to citrate fermentation. Also unique to strain P1059, but present in strains 36950, 3480, and HN06, are four genes involved in xylose $\mathrm{ABC}$ transport system with a transcriptional repressor (01538 to 01541).
Present in strains X73 and 36950 was a putative toxinantitoxin system similar to the HipAB systems (genes 02005 and 02006). Finally, genes for several novel proteins with similarity to the previously described Pfh-type filamentous hemagglutinins were identified in strains P1059 and X73. Strain P1059 contained a novel predicted filamentous hemagglutinin (designated PfhB4 gene \# 00523) that shares similarity with PfhB1 and PfhB2 from P. multocida. PfhB4 has conserved domains related to hemagglutination activity, two-partner secretion, hemagglutinin repeats, and toxicity. PfhB4 is present only in strains P1059, HN06, and 3480 (Figure 3). It is adjacent to a putative hemolysin secretion/activation protein (gene \# 00522) and thus appears to represent a novel two-partner system involved in hemagglutination. PfhB2 of strain P1059 has been shown to play an important role in either colonization or invasion in the turkey model [34]. Also, vaccination with recombinant P1059 PfhB2 peptides cross protected 


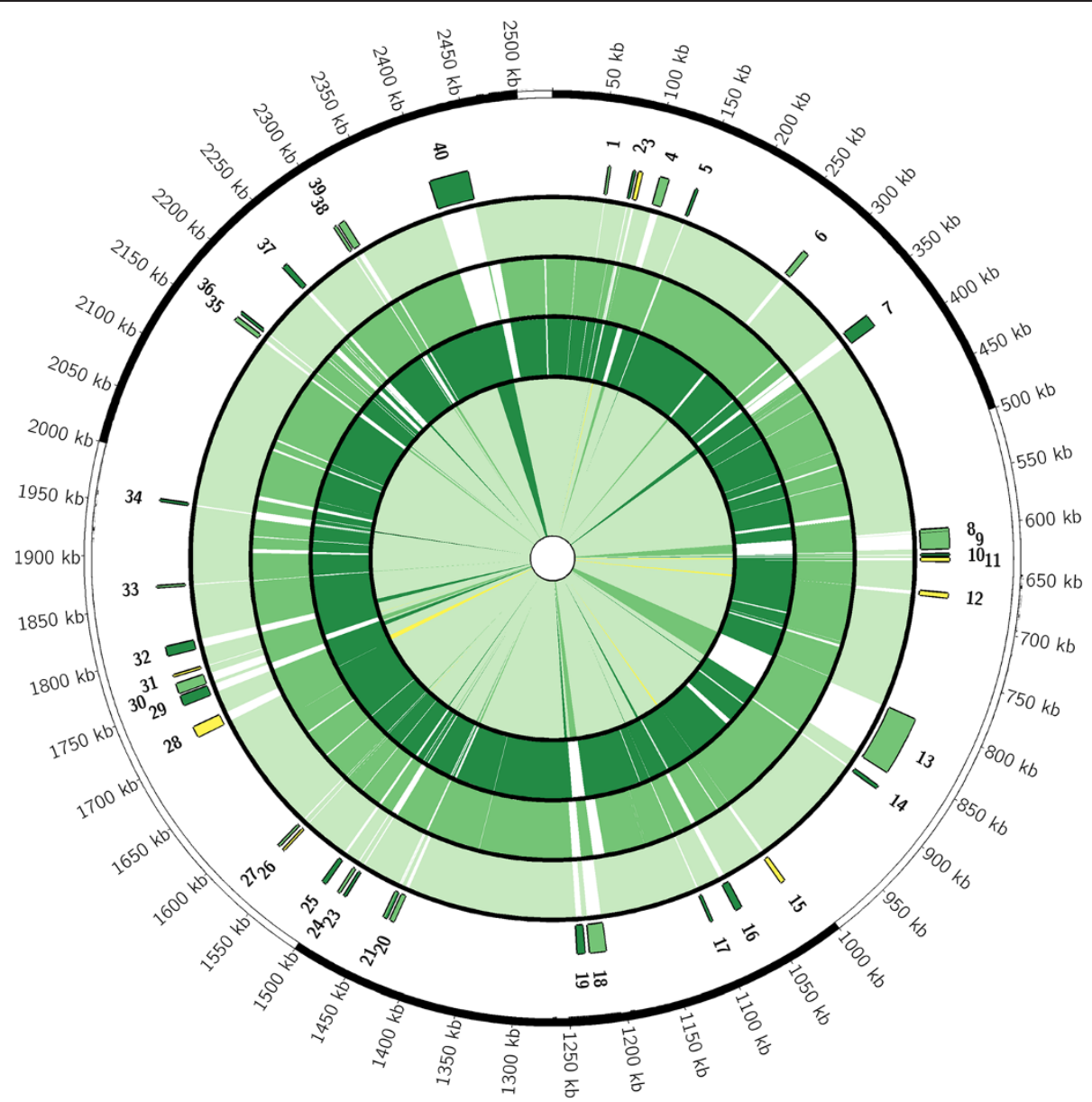

Figure 2 Circular map comparing sequenced avian source $P$. multocida strains. Scale is presented in $\mathrm{kb}$. The outermost rings depict genomic regions not present in strain Pm70 but present in strains P1059 (light green), X73 (dark green), or both (yellow). Regions are numbered as described in the Tables. The next three rings depict the shared genomic regions of avian source strains Pm70 (outer ring), P1059 (middle ring), and X73 (inner ring). Colored regions depict regions present at greater than $90 \%$ nucleotide similarity and non-colored regions depict regions absent. The innermost ring again depicts the core (very light green) regions present in all three strains and the regions absent from strain Pm70 but present in other sequenced strains using the same color scheme.

turkeys against an X73 challenge [35]. PfhB2 was present in strain Pm70, P1059, and X73, but was only 90\% similar in the latter two as compared to Pm70. Overall, the presence of unique genes/systems related to metabolism and adhesion could provide strains such as P1059 with additional tools for increased fitness leading to higher virulence.

Of the 127 unique proteins identified in strain X73 were five genes for a galactitol-specific phosphotransferase and utilization system (00310 to 00316), only present in strain X73; three genes for a TRAP dicarboxylate transporter system (01441 to 01443 ), also present in strain 36950; and six genes for a novel simple sugar D-allose transport and utilization systems (00951 to 00956), only present in strain X73. Such systems could again provide additional means of energy production in a resource-limited environment.

\section{Known virulence factors and antigens}

Comparisons were performed for several known virulence factors and outer membrane proteins that are important for P. multocida pathogenesis, functionality, and vaccine development [52]. These comparisons revealed some noteworthy aspects relative to their presence and evolution in $P$. multocida. For example, the hemoglobin receptors $h g b A$ and $h g b B$ were present in all sequenced $P$. multocida genomes, but are significantly different in their amino acid similarities (Table 3). HgbA and HgbB have been shown to exhibit hemoglobin binding properties $[53,54]$. Their incomplete distribution reported in previous studies could be attributed to genetic variation rather than complete absence of these genes [55]. The outer membrane porins $о m p H 1$ and $o m p H 2$ were also present in all sequenced strains, with ompH2 more highly conserved than ompH1 with respect to amino 
Table 2 Predicted proteins of interest present in $P$. multocida strains X73 and P1059 at greater than $90 \%$ similarity but present at less than $\mathbf{9 0 \%}$ similarity in strain Pm70

\begin{tabular}{|c|c|c|}
\hline $\begin{array}{l}\text { Gene } \\
\text { locus }\end{array}$ & $\begin{array}{l}\text { Length } \\
\text { (aa) }\end{array}$ & Predicted function \\
\hline 00056 & 576 & Hemolysin activator protein precursor \\
\hline 00060 & 1767 & $\begin{array}{l}\text { Exoprotein involved in heme utilization or } \\
\text { adhesion - PfhB3 }\end{array}$ \\
\hline 00219 & 96 & Hypothetical protein \\
\hline 00361 & 617 & $\begin{array}{l}\text { Outer membrane iron receptor protein-Fe } \\
\text { transport }\end{array}$ \\
\hline 00444 & 80 & Hypothetical protein \\
\hline 00514 & 116 & Hypothetical protein \\
\hline 00515 & 91 & Hypothetical protein \\
\hline 00522 & 70 & Hypothetical protein \\
\hline 00795 & 972 & Beta-1,3-glucosyltransferase \\
\hline 01068 & 197 & $\begin{array}{l}\text { Opacity family integral membrane protein-Opa } \\
\text { protein }\end{array}$ \\
\hline 01069 & 169 & SprT- protein \\
\hline 01350 & 424 & Nucleoside permease -NupC \\
\hline
\end{tabular}

acid similarity. Furthermore, a third outer membrane porin $о m p H 3$ was present in all sequenced strains except strain X73, but was highly conserved within these strains. The ptfA gene, encoding a type 4 fimbrial subunit, was highly conserved in all sequenced strains, as was $\operatorname{comE}$ encoding a fibronectin-binding protein. The pfhB1 gene, encoding a filamentous hemagglutinin protein, was present in strains Pm70, P1059, X73, and 3480.

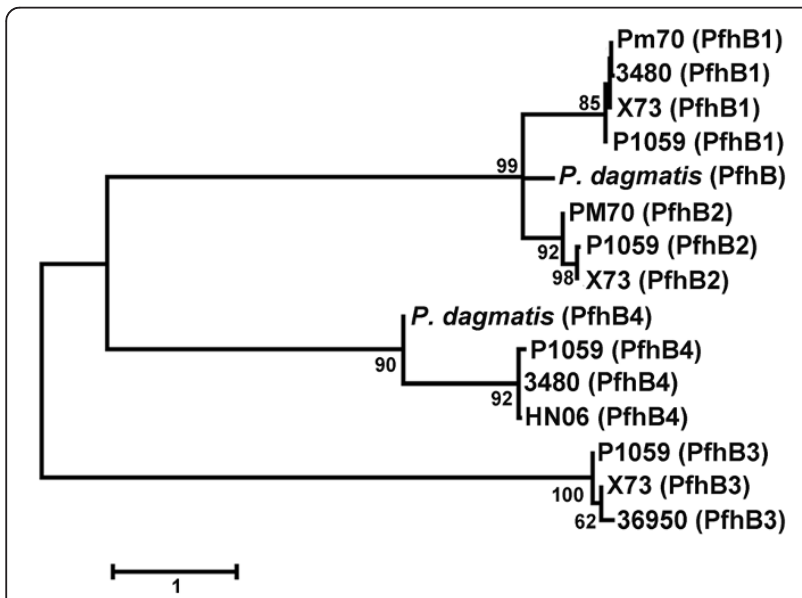

Figure 3 Dendrogram depicting amino acid sequence similarities between the filamentous heagglutinins of Pasteurella multocida. Evolutionary history was inferred using the Maximum Likelihood method based on the JTT matrix-based model. The tree is drawn to scale, and 500 bootstrap iterations were performed. A total of 1,479 positions were used in the final dataset. The analyses were conducted in MEGA [Tamura et al. 2007]. Proteins from $P$. dagmatis were included for comparative purposes.
Table 3 Similarity of proteins of interest in sequenced avian Pasteurella multocida genomes

\begin{tabular}{lllllll}
\hline Protein name & Pm70 & P1059 & X73 & $\mathbf{3 6 9 5 0}$ & HN06 & $\mathbf{3 4 8 0}$ \\
\hline HgbA & $100^{A}$ & 87 & 96 & 89 & 99 & 99 \\
HgbB & 100 & - & 95 & - & 84 & - \\
Omp16 & 100 & 100 & 100 & 99 & 100 & 100 \\
OmpH1 & 100 & 84 & 83 & 83 & 84 & 99 \\
OmpH2 & 100 & 98 & 98 & 99 & 98 & 97 \\
OmpH3 & 100 & 97 & - & 98 & 97 & 98 \\
TbpA & 100 & 99 & 99 & 98 & 100 & 99 \\
PtfA & 100 & 100 & 100 & 100 & 100 & 99 \\
ComE & 100 & 99 & 100 & 99 & 99 & 99 \\
PlpE & 100 & 94 & 94 & - & - & - \\
PlpP & 100 & 84 & 82 & 98 & 72 & 76 \\
PlpB & 100 & 99 & 100 & 99 & 100 & 100 \\
PlpD & 100 & 100 & 100 & 100 & 100 & 100 \\
PfhB1 (PM0057) & 100 & 99 & 98 & - & - & 99 \\
PfhB2 (PM0059) & 100 & 90 & 90 & 97 & - & - \\
PfhB3 & - & $100^{B}$ & 98 & 96 & - & - \\
PfhB4 & - & 100 & - & - & 93 & 93 \\
\hline APrcen amis & & & & 99
\end{tabular}

${ }^{A}$ Percent amino acid similarity to same protein from strain Pm70.

${ }^{B}$ Percent amino acid similarity to same protein from strain P1059.

PfhB1 was highly conserved among these strains. PfhB2, a second filamentous hemagglutinin, was present in strains Pm70, P1059, X73, and 36950. This protein was more variable in amino acid sequence among these strains (Figure 3). Two other genes encoding filamentous hemaggultinins, $p f h B 3$ and $p f h B 4$, were absent in strain Pm70, with $p f h B 3$ present in strains P1059, X73, and 36950, and pfhB4 present in strains P1059, HN06, and 3480. Finally, lipoproteins $p l p P$, $p l p B$, and $p l p D$ were present in all sequenced strains, and all were highly conserved except plpP, whose product shared only $82-98 \%$ amino acid similarity between strains.

\section{Single nucleotide polymorphisms}

The three avian source $P$. multocida genomes were also compared for SNPs within the conserved regions of their genomes using MAUVE [42], and the SNPs were analyzed for their coding effects using SNPeff [44] (Table 4). A total of 31,021 SNPs were identified between strains Pm70 and P1059, and 26,705 SNPs were identified between strains Pm70 and X73. The density of SNPs varied considerably across the $P$. multocida genome, with some regions containing a much higher density of SNPs than the rest of the core genome (Figure 4). This suggests that some regions of the genome are under diversifying selection, while the majority of the genome is under neutral or purifying selection. The ratio between nonsynonymous to synonymous substitutions $(\mathrm{dN} / \mathrm{dS})$ is commonly used as a measure of purifying versus 
Table 4 Single nucleotide polymorphism (SNP) analysis and dN/dS ratios of categorized and selected coding regions of Pasteurella multocida strains Pm70, P1059, and X73

\begin{tabular}{|c|c|c|c|c|}
\hline & Location & Non-synonymous & Synonymous & $\mathrm{dN} / \mathrm{dS}$ \\
\hline \multirow[t]{6}{*}{ Pm70 vs. P1059 } & Total & 8910 & 22111 & 0.4 \\
\hline & Cytoplasmic & 2431 & 9933 & 0.25 \\
\hline & Cytoplasmic membrane & 1556 & 5556 & 0.28 \\
\hline & Extracellular & 94 & 103 & 0.91 \\
\hline & Outer membrane & 1575 & 2062 & 0.76 \\
\hline & Periplasmic & 93 & 549 & 0.17 \\
\hline \multirow[t]{7}{*}{ Pm70 vs. $X 73$} & Total & 7401 & 19304 & 0.38 \\
\hline & Cytoplasmic & 2384 & 9162 & 0.26 \\
\hline & Cytoplasmic membrane & 1251 & 4710 & 0.27 \\
\hline & Extracellular & 125 & 134 & 0.93 \\
\hline & Outer membrane & 1783 & 1976 & 0.9 \\
\hline & Periplasmic & 98 & 593 & 0.17 \\
\hline & Function & Non-synonymous & Synonymous & $\mathrm{dN} / \mathrm{dS}$ \\
\hline PfhR (pm0040) & Putative porin-Fe transport & 7 & 15 & 0.47 \\
\hline PfhB1 (pm0057) & Filamentous hemagglutinin & 34 & 65 & 0.52 \\
\hline PfhB2 (pm0059) & Filamentous hemagglutinin & 498 & 506 & 0.98 \\
\hline Est (pm0076) & Outer membrane esterase & 39 & 59 & 0.66 \\
\hline PtfA (pm0084) & Type IV fimbrial subunit-ptfA & 4 & 0 & 4 \\
\hline HgbA (pm0300) & TonB-dependent hemoglobin receptor & 159 & 152 & 1.05 \\
\hline Csy1 (pm0305) & CRISPR-associated protein & 290 & 130 & 2.23 \\
\hline OmpW (pm0331) & Outer membrane protein & 2 & 4 & 0.5 \\
\hline pm0336 & TonB-dependent receptor & 39 & 57 & 0.68 \\
\hline HgbB (pm0337) & Hemoglobin binding protein & 78 & 90 & 0.87 \\
\hline OmpH_1 (pm0388) & Outer membrane porin & 36 & 66 & 0.55 \\
\hline OmpH_2 (pm0339) & Outer membrane porin & 10 & 16 & 0.63 \\
\hline TolC1 (pm0527) & Outer membrane efflux channel & 12 & 44 & 0.27 \\
\hline Pcp (pm0554) & Peptidoglycan-associated protein & 0 & 3 & 0 \\
\hline HemR (pm0576) & Hemoglobin binding receptor & 6 & 4 & 1.5 \\
\hline pm0591 & Secreted effector protein & 75 & 40 & 1.88 \\
\hline PhyA (pm0773) & Capular polysacharride export protein & 2 & 4 & 0.5 \\
\hline OmpA (pm0786) & Outer membrane protein & 61 & 70 & 0.87 \\
\hline Pm0803 & Outer membrane receptor protein, mostly Fe transport & 67 & 58 & 1.16 \\
\hline TadF (pm0844) & Pilus assembly protein & 112 & 81 & 1.38 \\
\hline TadE (pm0845) & Pilus assembly protein & 134 & 70 & 1.91 \\
\hline TadD (pm0846) & Pilus assembly protein & 126 & 103 & 1.22 \\
\hline RcpB (pm0851) & Pilus assembly protein & 144 & 69 & 2.08 \\
\hline RcpA (pm0852) & Pilus assembly protein & 182 & 222 & 0.82 \\
\hline RcpC (pm0853) & Pilus assembly protein & 166 & 112 & 1.48 \\
\hline Flp1 (pm0855) & Flp pilin component & 21 & 19 & 1.11 \\
\hline pm0998 & Hypothetical protein & 6 & 4 & 1.5 \\
\hline NanB (pm1000) & Outer membrane sialydase & 157 & 161 & 0.98 \\
\hline TonB (pm1188) & TonB energy supply via iron transport & 3 & 4 & 0.75 \\
\hline GlpQ (pm1444) & Glycerophosphodiester & 2 & 3 & 0.67 \\
\hline
\end{tabular}


Table 4 Single nucleotide polymorphism (SNP) analysis and dN/dS ratios of categorized and selected coding regions of Pasteurella multocida strains Pm70, P1059, and X73 (Continued)

\begin{tabular}{|c|c|c|c|c|}
\hline PlpE (pm1517) & Protective outer membrane lipoprotein & 24 & 39 & 0.62 \\
\hline PlpP (pm1518) & Protective outer membrane lipoprotein & 63 & 55 & 1.13 \\
\hline TorD (pm1794) & Chaperone & 4 & 3 & 1.33 \\
\hline
\end{tabular}

diversifying selection [56]. The overall $\mathrm{dN} / \mathrm{dS}$ ratios of all coding regions of strains P1059 and X73 compared to strain Pm70 were 0.40 and 0.38 , respectively. Proteins were then divided into groups based upon predicted subcellular localization of each protein using PSORT-B version 3.0. Using this approach, the $\mathrm{dN} / \mathrm{dS}$ ratios varied considerably, with higher ratios $(0.76-0.93)$ found within proteins predicted as extracellular or outer membrane [57]. Amongst specific outer membrane proteins, the highest $\mathrm{dN} / \mathrm{dS}$ ratios were observed within PfhB2, HgbA, HemR, pm0591 (a secreted effector protein), pm0803 (an iron-regulated outer membrane protein), TadD-F (pilus assembly proteins), RcpB-C (pilus assembly proteins), and PlpP. The higher $\mathrm{dN} / \mathrm{dS}$ ratios observed among this subset of extracellular and outer membrane proteins is suggestive that they are under diversifying selection due to interactions with the host immune system, although further analyses would be required to confirm this observation.

\section{LPS genes}

The Heddleston somatic typing system classifies $P$. multocida into 16 somatic types based on antigenic differences in the lipopolysaccharide (LPS) [6]. Good progress has been made in understanding the structural basis for the LPS typing scheme. The genes and the transferases required for the biosynthesis of the somatic type-specific outer core region of the LPS has been identified in strains of $P$. multocida strains representing various somatic types [24,58-63]. Since endotoxin (LPS) is a key virulence factor in $P$. multocida, we examined each gene involved in LPS biosynthesis in the X73 and P1059 strains and compared with the Pm70 strain. All three strains produced two glycoforms simultaneously, termed glycoforms A and B. Both X73 and P1059 contained the inner core biosynthetic complement of genes, including kdtA (P1059-01455; X73- 01363), hptA (opsX; P1059-02017; X73- 01921), kdkA (P1059-01451; X73-01359), hptC (rfaF; P1059-02018; X73-01922), hptD (P1059-01443; X73-01351) and gctA (P1059-01456; X7301364). The gene that encodes for the enzyme which catalyzes the attachment of phosphoethanolamine to L- $\alpha-D$ Heptose -11 (Pm70-pm0223) was present only in strains P1059 and Pm70. There appeared to be some variation in the $h p t D$ gene between Pm70 and the X73 and P1059 strains although it was generally conserved between strains. Linking the inner core to the outer core is the hptE gene, present in both X73 and P1059 (X73-01185; P1059-01293). The outer core structure expressed by X73, P1059 and Pm70 strains are structurally distinct and distal part of the molecule because in all three strains a polymeric $\mathrm{O}$ antigen was absent. The X73 strain but not P1059 and Pm70 express an outer core oligosaccharide that contains two terminal galactose residues, with phosphocholine (PCho). Present in X73 but absent from Pm70 and P1059 were the outer core biosynthetic genes involved in phosphocholine (PCho) biosynthesis genes for somatic type 1. As reported previously [23], these

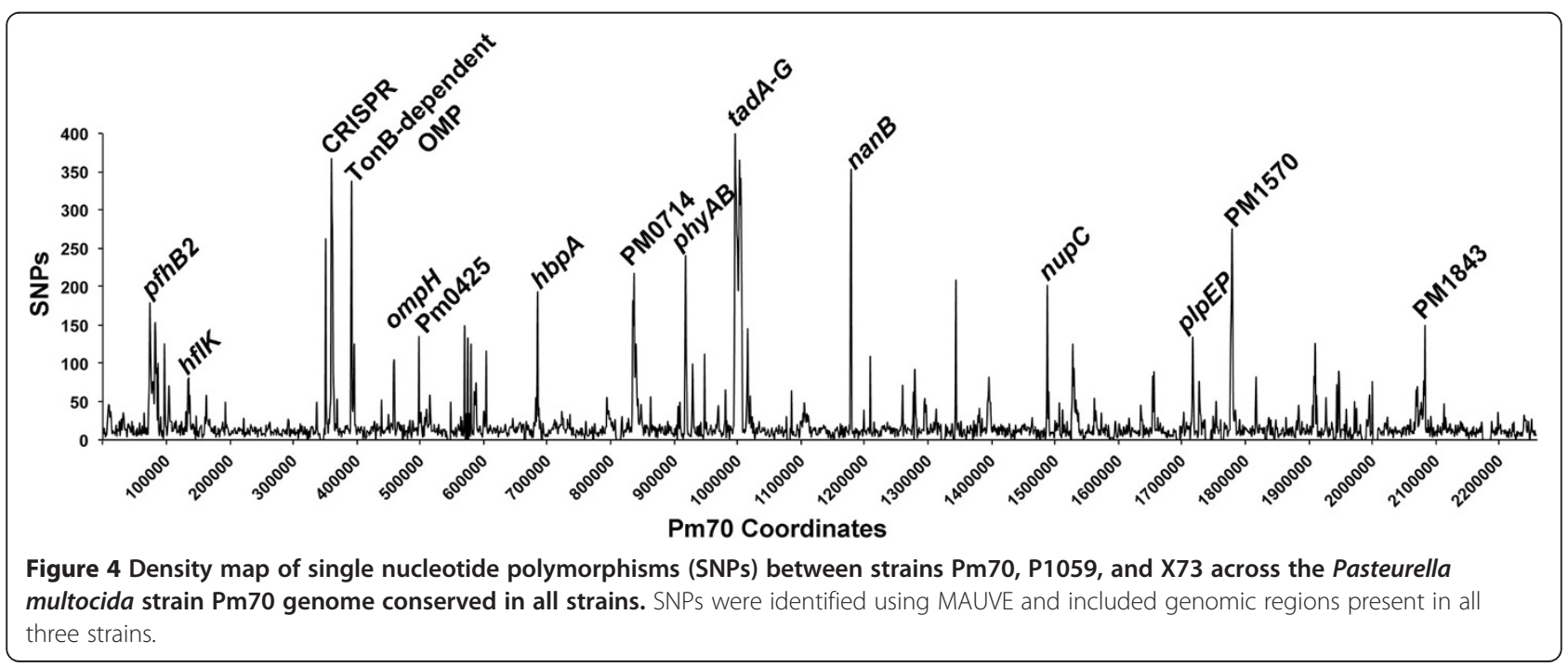


genes include $p c g A$ (X73-01180), $p c g B$ (X73-01182), $p c g C$ (X73-01181), and $p c g D$ (X73-01183) as well as gatA (X73-01184). X73 attaches a phosphoethanolamine (PEtn) residue to the terminal galactose. Studies have shown [23] that PCho on the LPS is important for virulence of X73 strain to chickens. However, a clear role for PEtn has not been defined. Present in the outer core of Pm70 and P1059, but absent in X73, were the biosynthetic genes for somatic type 3. These genes include losA (Pm70Pm1143; P1059-01292); (Pm70-Pm1138; P1059-01287); (Pm70-Pm1139; P1059-01288); (Pm70-Pm1140; P105901289); and (Pm70- Pm1141; P1059-01290).

In summary, comparative analyses of highly virulent versus avirulent $P$. multocida identified a number of genomic differences that may shed light on the ability of highly virulent strains to cause disease in the avian host. Most of the differences observed involved the presence of additional systems in virulent avian-source strains P1059 and/or X73 that appear to play metabolic roles. Such systems might enhance the fitness of these strains in the avian extraintestinal compartment, but without experimental evidence this is purely a speculative observation. This work does, however, underscore the need to utilize such genomic data towards targeted molecular approaches to better understand the role of horizontal gene transfer in the pathogenesis of this organism. Also, it is evident given the high degree of large sequence and single nucleotide polymorphisms in P. multocida that focused studies need to be conducted to appreciate adaptation of these strains to their respective hosts.

\section{Additional files}

Additional file 1: Table S1. Coding regions present in Pasteurella multocida strain P1059 but absent from strains Pm70 and X73, excluding prophage-associated regions.

Additional file 2: Table S2. Coding regions present in Pasteurella multocida strain X73 but absent from strains Pm70 and P1059, excluding prophage-associated regions.

\section{Competing interests}

The authors declare that they have no competing interests.

\section{Authors' contributions}

TJJ performed the genomic analysis, and was the primary author of this study. JEA participated in bioinformatics analyses, including sequence annotation, alignments and pathway reconstruction. SSH formatted and prepared assemblies and annotations for submission to GenBank. MH was involved in analyzing the genome sequences. FMT participated in the editorial review of the manuscript. SKM coordinated this study and helped to draft the manuscript. REB conceived this study, performed the genome sequences data and participated in writing of the manuscript. All authors read and approved the final manuscript.

\section{Acknowledgements}

This work was supported by the Biotechnology Research and Development Corporation, Peoria, Illinois, USA. Tools for comparative genome analysis were provided through support of the Minnesota Supercomputing Institute.

\section{Author details}

'Department of Veterinary and Biomedical Sciences, University of Minnesota, St. Paul, Minnesota, USA. ${ }^{2}$ Institute for Bioinformatics and Evolutionary Studies, University of Idaho, Moscow, Idaho, USA. ${ }^{3}$ National Animal Disease Center, Agricultural Research Service, US Department of Agriculture, Ames, lowa, USA.

Received: 2 January 2013 Accepted: 6 May 2013

Published: 14 May 2013

\section{References}

1. Christensen JP, Bisgaard M: Avian pasteurellosis: taxonomy of the organisms involved and aspects of pathogenesis. Avian Path 1997, 26:461-483.

2. Christenson JP, Bisgaard M: Fowl Cholera. Rev Sci Tech 2000, 19:626-637.

3. Wilkie IW, Harper M, Boyce JD, Adler B: Pasteurella multocida: Diseases and Pathogenesis. Curr Top Microbiol Immunol 2012, 361:1-22.

4. Carter GR: Studies on Pasteurella multocida. A hemagglutination test for the identification of serological types. Amer J Vet Res 1955, 16:481-484.

5. Carter GR: A new serological type of Pasteurella multocida from Central Africa. Vet Rec 1961, 73:1052.

6. Heddleston KL, Gallagher JE, Rebers PA: Fowl cholera: Gel diffusion precipitin test for serotyping Pasteurella multocida from avian species. Avian Dis 1972, 16:925-936.

7. Carter GR, Chengappa MM: Recommendations for a standard system of designating serotypes of Pasteurella multocida. Proceedings of the 24th Amer. Assoc. Veterinary Laboratory Diagnosticians 1981, 24:37-42.

8. Rhodes KR, Rimler RB: Somatic serotypes of Pasteurella multocida strains isolated from avian hosts (1976-1988). Avian Dis 1990, 34:193-195.

9. Lee MD, Wooley RE, Glisson JR, Brown J: Comparison of Pasteurella multocida serotype 3,4 isolates from turkeys with fowl cholera. Avian Dis 1988, 32:501-508.

10. Webster LT: The epidemiology of fowl cholera. J Exp Med 1930, 51:219-223.

11. Petersen KD, Christensen JP, Permin A, Bisgaard M: Virulence of Pasteurella multocida subsp. multocida isolated from outbreaks of fowl cholera in wild birds for domestic poultry and game birds. Avian Pathol 2001, 30:27-31.

12. Heddleston KL, Rebers PA: Properties of free endotoxin from Pasteurella multocida. Am J Vet Res 1975, 36:573-574.

13. Rhodes KR, Rimler RB: Effect of Pasteurella multocida endotoxins on turkey poults. Avian Dis 1987, 31:523-526.

14. Heddleston KL, Rebers PA, Ritchie AE: Immunizing and toxic properties of particulate antigens from two immunogenic types of Pasteurella multocida of avian origin. J Immunol 1966, 96:124-133.

15. May BJ, Zhang Q, Li LL, Paustian ML, Whitman TS, Kapur V: Complete genome sequence of Pasteurella multocida Pm70. Proc Natl Acad Sci USA 2001, 98:3460-3465.

16. Steenbergen SM, Lichtensteiger CA, Caughlan R, Garfinkle J, Fuller TE, Vimr ER: Sialic acid metabolism and systemic pasteurellosis. Infect Immun 2005, 73:1284-1294

17. Steen JA, Steen JA, Harrison P, Seemann T, Wilkie I, Harper M, Adler B, Boyce JD: Fis is essential for capsule production in Pasteurella multocida and regulates expression of other important virulence factors. PLoS Pathog 2010, 6:e1000750.

18. Nanduri B, Shack LA, Burgess SC, Lawrence ML: The transcriptional response of Pasteurella multocida to three classes of antibiotics. BMC Genomics 2009, 14(10 Suppl 2):S4.

19. Boyce JD, Wilkie L, Harper M, Paustian ML, Kapur V, Adler B: Genomic scale analysis of Pasteurella multocida gene expression during growth within liver tissue of chickens with fowl cholera. Microbes Infect 2004, 6:290-298.

20. Paustian ML, May BJ, Kapur V: Transcriptional response of Pasteurella multocida to nutrient limitation. J Bacteriol 2002, 184:3734-3739.

21. Nanduri B, Lawrence ML, Peddinti DS, Burgess SC: Effects of subminimum inhibitory concentrations of antibiotics on the Pasteurella multocida proteome: a systems approach. Comp Funct Genomics 2008. doi:10.1155/ 2008/254836.

22. E-Komon T, Burchmore R, Herzyk P, Davies R: Predicting the outer membrane proteome of Pasteurella multocida based on consensus prediction enhanced by results integration and manual confirmation. BMC Bioinformatics 2012, 13:63-80. 
23. Harper M, Cox A, St Michael F, Parnas H, Wilkie I, Blackall PJ, Adler B, Boyce JD: Decoration of Pasteurella multocida lipopolysaccharide with phosphocholine is important for virulence. J Bacterio/ 2007, 189:7384-7391.

24. St Michael F, Vinogradov E, Li J, Cox AD: Structural analysis of the lipopolysaccharide from Pasteurella multocida genome strain Pm70 and identification of the putative lipopolysaccharide glycosyltransferases. Glycobiology 2005, 15:323-333.

25. Bosch M, Garrido ME, de Rozas AM P, Badiiola I, Barbe J, Llagostera M: Pasteurella multocida contains multiple immunogenic haemin- and haemoglobin-binding proteins. Vet Microbio/ 2004, 99:102-112.

26. Hatfaludi T, Al-Hasani K, Gong L, Boyce JD, Ford M, Wilkie IW, Quinsey N, Dunstone MA, Hoke DE, Adler B: Screening of $71 P$. multocida proteins for protective efficacy in a fowl cholera infection model and characterization of the protective antigen PIpE. PLoS One 2012, 7:e39973.

27. Ewers C, Becker AL, Bethe A, Kiebling S, Filter M, Wieler LH: Virulence genotype of Pasteurella multocida strains isolated from different hosts with various disease status. Vet Microbiol 2006, 114:304-317.

28. Al-Hasani K, Boyce J, McCarl VP, Bottornley S, Wilkie I, Adler B: Identification of novel immunogens in Pasteurella multocida. Microb Cell Fact 2007, 6:1-5.

29. Rhoades KR: The microscopic lesions of acute fowl cholera in mature chickens. Avian Dis 1964, 8:658-665.

30. Heddleston KL: Studies on pasteurellosis. V. Two immunogenic types of Pasteurella multocida associated with fowl cholera. Avian Dis 1962, 6:315-321.

31. Boyce JD, Seemann T, Adler B, Harper M: Pathogenomics of Pasteurella multocida. Curr Top Microbiol Immunol 2012, 361:23-38.

32. Boyce JD, Harper M, Wilkie IW, Adler B: Pasteurella. In Pathogenesis of Bacterial Infections in Animals. Chapter 17. 4th edition. Edited by Gyles CL, Prescott JF, Songer JG, Thoen CO. Ames, lowa: Wiley-Blackwell; 2010.

33. Lee MD, Wooley RE, Brown J, Glisson JR: A survey of potential virulence markers from avian strains of Pasteurella multocida. Vet Microbiol 1991 26:213-225

34. Tatum FM, Yersin AG, Briggs RE: Construction and virulence of a Pasteurella multocida fhaB2 mutant in turkeys. Microb Pathog 2005, 39:9-17.

35. Tatum FM, Tabatabai LB, Briggs RE: Cross-protection against fowl cholera disease with the use of recombinant Pasteurella multocida FHAB2 peptides. Avian Dis 2012, 56:589-591.

36. Kumar S, Blaxter ML: Comparing de novo assemblers for 454 transcriptome data. BMC Genomics 2010, 11:571.

37. Besemer J, Lomsadze A, Borodovsky M: GeneMarkS: a self-training method for prediction of gene starts in microbial genomes. Implications for finding sequence motifs in regulatory regions. Nucleic Acids Res 2001, 29:2607-2618

38. Altschul SF, Madden TL, Schaffer AA, Zhang J, Zhang Z, Miller W, Lipman DJ: Gapped BLAST and PSI-BLAST: a new generation of protein database search programs. Nucleic Acids Res 1997, 25:3389-3402.

39. McClure MA, Smith C, Elton P: Parameterization studies for the SAM and HMMER methods of hidden Markov model generation. Proc Int Conf Intell Syst Mol Biol 1996, 4:155-64.

40. Lowe TM, Eddy SR: tRNAscan-SE: a program for improved detection of transfer RNA genes in genomic sequence. Nucleic Acids Res 1997, 25:955-964.

41. Lagesen $K$, Hallin $P$, Rodland EA, Staerfeldt HH, Rognes T, Ussery DW: RNAmmer: consistent and rapid annotation of ribosomal RNA genes. Nucleic Acids Res 2007, 35:3100-3108.

42. Darling AC, Mau B, Blattner FR, Perna NT: Mauve: multiple alignment of conserved genomic sequence with rearrangements. Genome Res 2004 14:1394-1403

43. Darzentas N: Circoletto: visualizing sequence similarity with Circos. Bioinformatics 2010, 26:2620-2621.

44. Cingolani P, Platts A, le Wang L, Coon M, Nguyen T, Wang L, Land SJ, Lu X, Ruden DM: A program for annotating and predicting the effects of single nucleotide polymorphisms, SnpEff: SNPs in the genome of Drosophila melanogaster strain w1118; iso-2; iso-3. Fly (Austin) 2012, 6(2):80-92.

45. Muraoka WT, Zhang Q: Phenotypic and genotypic evidence for L-fucose utilization by Campylobacter jejuni. J Bacteriol 2011, 193:1065-1075.

46. Stahl M, Friis LM, Nothaft H, Liu X, Li J, Szymanski CM, Stintzi A: L-fucose utilization provides Campylobacter jejuni with a competitive advantage. Proc Natl Acad Sci USA 2011, 108:7194-7199.

47. Ahir VB, Roy A, Jhala MK, Bhanderi BB, Mathakiya RA, Bhatt VD, Padiya KB, Jakhesara SJ, Koringa PG, Joshi CG: Genome sequence of Pasteurella multocida subsp. gallicida Anand1_poultry. J Bacteriol 2011, 193:5604.
48. Michael GB, Kadlec K, Sweeney MT, Brzuszkiewicz E, Liesegang H, Daniel R, Murray RW, Watts JL, Schwarz S: ICEPmu1, an integrative conjugative element (ICE) of Pasteurella multocida: structure and transfer. J Antimicrob Chemother 2012, 67:91-100.

49. Liu W, Yang M, Xu Z, Zheng H, Liang W, Zhou R, Wu B, Chen H: Complete genome sequence of Pasteurella multocida HN06, a toxigenic strain of serogroup D. J Bacteriol 2012, 194:3292-3293.

50. Muhairwa AP, Christensen JP, Bisgaard M: Investigations on the carrier rate of Pasteurella multocida in healthy commercial poultry flocks and flocks affected by fowl cholera. Avian Pathol 2000, 29:133-142.

51. Christensen $H$, Bisgaard M, Bojesen AM, Mutters R, Olsen JE: Genetic relationships among avian isolates classified as Pasteurella haemolytica, Actinobacillus salpingitidis' or Pasteurella anatis with proposal of Gallibacterium anatis gen. nov., comb. nov. and description of additional genomospecies within Gallibacterium gen. nov. Int J Syst Evol Microbiol 2003, 53(Pt 1):275-87.

52. Hatfaludi T, Al-Hasani K, Boyce JD, Adler B: Outer membrane proteins of Pasteurella multocida. Vet Microbiol 2010, 14:1-17.

53. Bosch M, Garrido ME, Llagostera M, Perez De Rozas AM, Badiola I, Barbe J: Characterization of the Pasteurella multocida hgbA gene encoding a hemoglobin-binding protein. Infect Immun 2002, 70:5955-64.

54. Cox AJ, Hunt ML, Boyce JD, Adler B: Functional characterization of HgbB, a new hemoglobin binding protein of Pasteurella multocida. Microb Pathog 2003, 34:287-96.

55. Garcia N, Fernandez-Garayzabal JF, Goyache J, Dominguez L, Vela Al: Associations between biovar and virulence factor genes in Pasteurella multocida isolates from pigs in Spain. Vet Rec 2011, 169:362.

56. Rocha EP, Smith JM, Hurst LD, Holden MT, Cooper JE, Smith NH, Feil EJ: Comparisons of $\mathrm{dN} / \mathrm{dS}$ are time dependent for closely related bacterial genomes. J Theor Biol 2006, 239:226-35.

57. Gardy JL, Spencer C, Wang K, Ester M, Tusnady GE, Simon I, Hua S, DeFays K, Lambert C, Nakai K, et al: PSORT-B: Improving protein subcellular localization prediction for Gram-negative bacteria. Nucleic Acids Res 2003, 31:3613-7.

58. Harper M, Cox AD, Adler B, Boyce JD: Pasteurella multocida lipopolysaccharide: The long and the short of it. Vet Microbiol 2011, 153:109-15.

59. Harper M, St Michael F, John M, Vinogradov E, Adler B, Boyce JD, Cox AD: Pasteurella multocida Heddleston serovars 1 and 14 express different lipopolysaccharide structures but share the the same lipopolysaccharide biosynthesis outer core locus. Vet Microbiol 2011, 150:289-96.

60. Harper M, St Michael F, Vinogradov E, John M, Boyce JD, Adler B, Cox AD: Characterization of the lipopolysaccharide from Pasteurella multocida Heddleston serovar 9; identification of a proposed bi-functional dTDP-3 -acetamido-3,6-dideoxy-a-D-glucose biosynthesis enzyme. Glycobiology 2012, 22:332-44.

61. St Michael F, Harper M, Parnas H, John M, Stupak J, Vinogradov E, Adler B, Boyce JD, Cox AD: Structural and genetic basis for the serological differentiation of Pasteurella multocida Heddleston serotypes 2 and 5. J Bacteriol 2009, 191:6950-59.

62. St Michael F, Li J, Cox AD: Structural analysis of the core oligosaccharide from Pasteurella multocida strain X73. Carbohydr Res 2005, 340:1253-57.

63. Harper M, St Michael F, Vinogradov E, John M, Steen JA, Van Dorsten L, Boyce JD, Adler B, Cox AD: Structure and biosynthetic locus of the lipopolysaccharide outer core produced by Pasteurella multocida serovars 8 and 13 and the identification of a novel phosphoglycero moity. Glycobiology 2013, 23:286-294.

\section{doi:10.1186/1471-2180-13-106}

Cite this article as: Johnson et al.: Comparative genome analysis of an avirulent and two virulent strains of avian Pasteurella multocida reveals candidate genes involved in fitness and pathogenicity. BMC Microbiology 2013 13:106. 